\title{
On recent speech corpora activities in $\operatorname{Japan}^{\dagger}$
}

\author{
Shuichi Itahashi \\ Institute of Information Sciences and Electronics, University of Tsukuba, \\ 1-1-1 Tennodai, Tsukuba, 305-8573 Japan
}

\begin{abstract}
This paper describes a range of Japanese projects which are concerned with speech corpora. ETL is to be credited for initiating research on speech database in 1973, while Tohoku University played a pioneering role in speech corpus development. The JEIDA Japanese Common Speech Data Corpus was reported in 1986 and then later converted to DAT form. Subsequently in 1990, the JEIDA Noise Database was released to the public. Other important contributions are due not only to ATR which has developed a wide variety of speech corpora, but also to the so-called priority area projects funded by MESSC. On the one hand, the "Spoken Language" project has yielded data on continuous speech, while the "Spoken Japanese" project yielded data on various dialectal speech from all over Japan. On the other hand, the "Spoken Dialogue" project has yielded data on various spoken dialogues. Six CD-ROMs were produced by a committee of the Acoustical Society of Japan. Three of them contain speech of isolated sentences that are phonetically balanced, while the remaining three include continuous speech obtained for various guide-tasks. This paper finally refers to the new ASJ corpus and the "Real World Computing Program" formulated in 1992 by the Japanese Government.
\end{abstract}

Keywords: Speech, Corpus, Database, Dialogue, Label

PACS number: $43.72 . \mathrm{Kb}$

\section{INTRODUCTION}

Although the necessity of having common speech data has long been acknowledged, the realization of such a data corpus has been slowly forthcoming.

In this vein, we have realized that it is necessary to create various kinds of language/speech corpora available for common use, and to establish a system for the research and development and the performance evaluation of various speech equipment. However, our efforts to implement this "concept" are still on a small scale and fragmented in Japan. It is a pressing need to coordinate our efforts, for the sake of academic research, international collaboration, and indutrial promotions.

Owing to the need to prepare a systematic, com-

+ A revised version of a paper (Itahashi, 1998b) presented at the First International Conference on Language Resources and Evaluation (LREC98) held in Granada, Spain, May 1998. mon framework to collect, create, store, distribute and share language/speech data for securing progress in future research, we have come to establish the Linguistic Resources Sharing Initiative (LRSI). Consisting of 23 members interested in language/ speech corpus, the LRSI held a preparatory meeting on March 1, and a symposium on June 30, 1994. However, there are serious concerns in regard to the availability of financial support.

At present, speech corpora accesible by the public in Japan are those of JEIDA (Japan Electronic Industry Development Association), ATR (Advaced Telecommunications Research Laboratories), ETL (Electrotechnical Laboratory), ASJ (Acoustical Society of Japan), Research Projects sponsored by MESSC (the Ministry of Education, Science, Sports and Culture), Tohoku University, and RWC (Real World Computing Partnership) Program. NTT (Nippon Telegraph and Telephone Corporation) and other private companies have 
developed their own speech corpora, which are however not available to the public.

This paper describes recent speech corpora projects in Japan in some of which the author has been involved (Itahashi, 1987, 1990, 1991a).

\section{PROGRESS OF SPEECH CORPORA WORK IN JAPAN}

\subsection{ETL : Electrotechnical Laboratory}

ETL initiated research on speech databases in Japan (Nakajima et al., 1973). It developed a speech database with labeling of each subphonemic unit.

The ETL Speech Databases include a set of 1,542 phonetically balanced words, and fine acousticphonetic-labels of speech samples (Tanaka et al., 1986, 1990). The word set has been chosen from a Japanese word dictionary (vocabulary size, 44,000 words) so that it contains all variations of threephoneme sequences, i.e., $\mathrm{VVV}, \mathrm{VCV},\langle\mathrm{CV}, \mathrm{CV}\rangle$, VQC, ${ }^{\dagger 1} \mathrm{CVC}, \mathrm{CVV}$ and VVC, which occurred in the dictionary and gave the maximal entropy for these sequences. The acoustic-phonetic labeling has been carried out by a semi-automatic labeling system (Tanaka et al., 1986). The labeling unit is an acoustically compact segment called APSEG. For example, phoneme sequence /-sum-/ is basically interpreted to "-SS-SU-UU-UM-MM-", but in real samples, some of which vary acoustically as "-SSSU-UM-MM-" (i.e., elimination of steady part of a vowel) or "-SS-SUM-MM-" (i.e., elimination of a vowel). Therefore, speech is represented in directed graphs. Several rules were obtained for network pattern variations from the labeled database, and incorporated to a large vocabulary word recognition system which yielded a high recognition score. The word set is available to the public, and, to date, has been used in several research divisions of companies in Japan. The labeled speech database is also available to the public, and is used at several research laboratories.

\subsection{Tohoku University}

Tohoku University played a pioneering role in speech corpus development. The Tohoku University Speech Database contains 12 GB speech wave data composed of a 212-word set, a 3,000-word set, a continuous speech set and an ATR speech data set

${ }^{\dagger 1} \mathrm{Q}$ denotes a geminate consonant, " $<$ " and " >" signify word boundary.
(Makino et al., 1990). These sets are stored on optical disks and CD-ROMs. The CD-ROMs contain the 212-word set and the 3,000-word set. A database management system is also provided, which includes an automatic labeling procedure, based on a given phoneme string of an input speech. The database itself is built up by making use of relational tables.

\subsection{JEIDA : Japan Electronic Industry Develop- ment Association}

Since 1982, a committee of 15 members from various Japanese research institutes and private companies have been involved in the development of speech corpora for common use, supported by JEIDA.

\subsubsection{JEIDA Japanese Common Speech Data Corpus (JCSD Corpus)}

The JEIDA Japanese Common Speech Data Corpus was reported by the author in 1986 for the first time. The initial version was recorded on video cassettes in PCM format. Later, a DAT version of the corpus was released with suitable start-IDs, making access much easier than before to necessary portions of the data.

The total data amount to 120 hours or 193,800 samples contained in 76 DAT cassettes. Speakers range from 20 to 60 years of age. The corpus is composed of 323 items with 4 utterances for each item spoken by 75 male and 75 female speakers, including 110 monosyllables, 178 isolated words and 35 4-digit sequences. The corpus has been distributed among 50 institutions in Japan. Further details are offered in (Itahashi, 1985, 1986).

The DATs have been converted recently to CDROMs, and are now distributed from LDC.

\subsubsection{The JEIDA Noise Database}

The Speech Input Systems Subcommittee of JEIDA collected various kinds of typical environmental noises, while giving consideration to environments where speech input devices are expected to be used. The noises are intended for use in performance assessment of speech recognizers.

Noise data were recorded on 18 DAT cassettes of 120 minutes, one of which is the digest version of the other 17 cassettes, yielding the so-called the JEIDA Noise Database. At the beginning of each tape, standard noise is recorded for sound level calibration, so that users can approximately reproduce the noise environment. Although it was expected that 


\section{S. ITAHASHI : ON RECENT SPEECH CORPORA ACTIVITIES IN JAPAN}

this database would include such noise environments as office rooms, train compartments, airplane cabins ; they were excluded for the sake of protecting privacy (Itahashi, 1990, 1991a, 1991d).

\subsection{ATR: Advanced Telecommunications Research Laboratory}

ATR Interpreting Telephony Research Laboratories started developing speech databases in 1986. The corpus comprises sets A, B, C, D, E, and F. Set A contains 8,500 isolated words uttered by 20 professional speakers. Set B comprises 503 phonetically-balanced sentences uttered by 12 speakers, with phonemic labeling. Set $\mathrm{C}$ consists of 750 words and 150 sentences spoken by 40 speakers. Set D contains various articles (about 400 sentences) uttered by two professional narrators, with grammatical tags. Sets $E$ and F comprise phonetically-balanced English and Japanese sentences.

The ATR database includes words, sentences, texts, spontaneous speech and noisy speech (Sagisaka et al., 1990; Takeda et al., 1987; Takezawa et al., 1998). For the sake of securing multiple purposes, five different transcriptions are given for almost all the databases, i.e., phonetic symbols, acoustic events, allophonic variants, inseparable portions, and vowel centers. Segment boundaries and transcriptions were made manually by visually inspecting sound spectrograms.

The isolated word database contains about 5,000 words extracted from a Japanese word dictionary, 216 phonemically balanced words, digits and about 100 Japanese syllables. They were uttered by 10 male and 10 female professional speakers. This database has been used at both ATR and universities.

The isolated sentence database consists of 503 sentences collected from newspapers and magazines. It contains all possible combinations of two phonemes with equal probability and as many combinations of three phonemes as possible. In this database, grammatical information such as part of speech, inflectional categories and phrasal structure is assigned to the constituents of the sentences. Up to now, 8 speakers have uttered this set, and their data have been used in the analysis of prosodic characteristics.

The mixture of word and sentence database was designed for the study of speaker-independent speech recognition tasks. It consists of about 750 words and 150 sentences.

The text speech database contains 11 articles (about 250 sentences) extracted from school text books and from NHK TV text books. They were uttered by two professional narrators, and grammatical information is also added to the sentences. Databases of spontaneous speech and noisy speech are also planned.

\subsection{MESSC Projects}

MESSC supports scientific research with several kinds of grants. Among them is the Grant-in-Aid for Scientific Research on Priority Areas. So far, three priority area projects on speech research have been carried out.

\subsubsection{Priority area project "Spoken Language"} and the DSR project

A research project on "Advanced Man-Machine Interface through Spoken Language" was carried out between 1987 and 1989 as one of the priority areas supported by the Grant-in-Aid for Scientific Research from the MESSC. The project considered a speech database as one of the important research themes (Fujisaki, 1987). The speech data were recorded on video cassettes through a PCM processor.

The contents of the corpus are as follows : Vowels and numerals (37 items), 216 Phonetically-balanced words (ATR list), 110 monosyllables, continuous speech of 80 short sentences and three texts. This corpus comprises two hours of speech per person, 10 male and 10 female speakers of 20 to 60 years of age; each item is uttered four times. The length of the data is 24 hours.

A further project funded by a Grant-in-Aid for Developmental Scientific Research on "Speech Database," has yielded conversion of the recorded data to DAT cassettes (Itahashi, 1990, 1991a). Part of the corpus was recorded on a CD-ROM in which each item is uttered once (Itahashi, 1992).

2.5.2 Priority area project: "Spoken Japanese"

A grant-in-Aid for Scientific Research on Priority Area "Spoken Japanese" project was carried out from fiscal 1989 to 1992 . The purpose of the project was to collect samples of various dialects spoken all over Japan, and to create speech databases, so that they can be used for linguistic, phonetic, acoustic and physiological analyses. The speech data will also be used for Japanese education of both 
Japanese and foreign students.

In particular, this project has yielded common speech items from 100 districts all over Japan. They contain about 1,450 items, including 1,300 word level items and 150 items of sentences and short stories.

Speech data were acquired using digital audio tape recorders (DAT). Part of the collected data has been edited and re-recorded onto DAT. Some of them have also been labeled and stored on CDs or CD-ROMs. This project yielded $19 \mathrm{CDs}$ and 3 CD-ROMs containing various Japanese dialects (Itahashi and Sugito, 1990; Itahashi, 1991b, 1992).

In sum, the "Spoken Japanese" corpus consists of Eastern Japanese dialects (5 CDs), Western Japanese dialects (6 CDs), Common items (2 CDs), the speech of AINU (1 CD), Ryukyu dialects (1 CD), Recitation by school children (1 CD), Japanese learners' speech (1 CD), and Folk tale and weather forecast (1 CD).

\subsubsection{Priority area project "Spoken Dialogue"}

In this section we refer to the "Simulated Spoken Dialogue Corpus." It was edited thanks to the support of the Grant-in-Aid for Scientific Research on Priority Areas Project "Research on Understanding and Generating Dialogue by Integrated Processing of Speech, Language and Concept," which was carried out from fiscal 1993 to 1995 . The resulting corpus contains the speech waves and their transcribed text of 93 simulated dialogues in Japanese on secretarial system, appointment scheduling, telephone shopping tasks and so on. The total length of time of dialogues is about $450 \mathrm{~min}$ utes. The basic specifications of the corpus were examined in the "Spoken Dialogue Corpus Working Group" made up of researchers from 11 universities in the project. The working group produced 4 CD-ROMs which contain all speech waves, transcribed texts and the HTML format files in which the transcribed utterances have links to speech wave files (Itahashi, 1998a).

\subsection{ASJ : Acoustical Society of Japan}

The committee for the Investigation of Continuous Speech Databases of the Acoustical Society of Japan was established in 1990. The members are primarily from universities, with some from national research institutes and private enterprises. The committee aims to investigate methods of designing continuous speech databases, and to propose tasks for the databases, by choosing suitable texts and programs for actual database creation. It has created continuous speech databases in mutual cooperation among its members (Itahashi, 1991c), and coordinates members' requests to utilize the databases.

\subsubsection{Continuous speech corpus}

The speech corpora are divided into two types. One is a collection of isolated sentences which are phonetically balanced. This corpus is composed of 503 sentences chosen by ATR Interpreting Telephony Research Laboratories. Three CD-ROMs were produced.

The other is a corpus which constitutes a story. For this purpose, samples of simulated conversational speech were first recorded onto DAT. They were transcribed into orthographic transcriptions, which were then re-written for more concise sentences. Various types of insertions were deleted, which included hesitation, interjection and dialectal expressions. Some phrases were added if necessary, otherwise we tried to keep the flavor of the original conversations. The next step was to read and record the re-written sentences as a story by one speaker. The scale of this corpus is almost the same as that of ATR PB-Sentences. This corpus was also recorded onto three CD-ROMs (Hayamizu et al., 1993).

The speech waves were digitized at $16 \mathrm{kHz}$ sampling frequency and quantized to 16 bits. The recording and $\mathrm{A} / \mathrm{D}$ conversion characteristics, including low-pass filter characteristics (flat up to 6 $\mathrm{kHz}$ and $-40 \mathrm{~dB}$ at $8 \mathrm{kHz}$ recommended), are not necessarily unified. The CD-ROM was created by the Japan Information Processing Development Center.

The ASJ Continuous Speech Corpus is composed of 7 CD-ROMs including ATR phoneticallybalanced sentences $(9,600$ sentences by 64 speakers), guide-task sentences $(12,000$ sentences by 36 speakers) and simulated dialogues ( 37 dialogues by 37 speakers).

\subsubsection{JNAS Corpus}

The newly created JNAS (Japanese Newspaper Article Read Speech Corpus) comprises 16 CDROMs (45,000 sentences uttered by 306 speakers). The JNAS corpus was created by the Acoustical Society of Japan in cooperation with the Information Processing Society of Japan (Itou et al., 1998). 
The Speech Database Committee of the Acoustical Society of Japan, and the Dictation Working Group of the Information Processing Society of Japan have discussed the design and creation of this corpus, which has been recorded in collaboration with 39 institutions including 19 universities and 19 private companies and a national research institute. The recording and A/D conversion characteristics, including low-pass filter characteristics, are not necessarily unified. The distribution of this corpus is restricted to the members of the ASJ.

This corpus contains orthographic transcriptions and speech recordings by 306 speakers (153 males and females each) reading excerpts from the Mainichi Newspaper and the ATR 503 PhoneticallyBalanced-Sentences. All utterances and sentences are in the Japanese language.

We prepared 155 text sets. Each set consists of about 100 sentences from the Mainichi Newspaper. As a general rule, each text set was read by one male and one female. Every speaker also read any subset of the ATR 503 PB-Sentences (about 50 sentences for each subset). That is, this corpus contains utterances of about 45,000 sentences as a whole with all speakers reading about 150 sentences each.

Each utterance was recorded with two microphones: a head-set microphone (all recoding sites used Sennheiser HMD410/HMD25-1 or the equivalent), and a desk-top microphone of different types at each site (Sanken, Sony, and so on). These two-microphone data were stored into separate files which have a similar directory structure in the CD-ROM directories; eight of the discs (Vol.1 through Vol.8) contain the head-set microphone data and the other (Vol.9 through Vol.16) the desktop microphone data.

The speech waves were sampled at $16 \mathrm{kHz}$ and quantized into 16 bits. They are stored in compressed format.

The corpus also includes orthographic transcriptions of the speech data and the bigram language. models for the Mainichi Newspaper articles from which the prompting text was selected.

\section{RWC PROGRAM}

In 1992 the Japanese government formulated a 10-year-program entitled the "Real World Computing Partnership," with a view to establishing new information processing systems.

Real World Computing aims to realize a flexible information system by introducing a human-like intuitive information processing capability. The main subjects in the technological development of the Real World Computing Program will be divided into the following two categories:

1) To establish the theoretical basis for and novel functions of "flexible information processing," based on advanced technologies endowed with human-like flexibility, learning capability and adaptability.

2) To develop information processing systems for processing large volumes of spatio-temporally distributed information at high speeds, while taking into account their interactions.

Subjects of Research and Development include Research on Theoretical Foundation, Novel Functions for Applications, Massively Parallel Systems, Neural Systems, and Optical Computing Systems.

The second theme of the above five subjects will include the following topics :

1) Flexible recognition and understanding of images, speech and natural language,

2) Flexible interface and problem solving, including flexible information bases and retrieval,

3) Flexible human interface and simulation,

4) Flexible autonomous control.

In relation to the first topic above, speech and text corpora have been collected and made available to researchers (Tanaka et al., 1996).

Two types of conversations were selected for the dialogue: one consists of dialogues between car dealers and customers, the other between travel agents and their customers. Professional dealers and agents were employed to add reality in the conversations. To date, 60 samples of dialogues have been recorded, 48 of which were filed into CD-ROMs including about 10 hours of speech waveforms with transcriptions and related-labeling. The dialogues are almost completely spontaneous but their acoustic quality is kept relatively high.

The RWC program also plans to create a read speech corpus for research on speech retrieval, topic extraction and utterance summarization. It will consist of read speech obtained from broadcasting manuscripts of financial news uttered by professional announcers. The texts for reading are selected from the recent actual news provided by Japan Broadcasting Corporation (NHK). The announcers are employed for this recording. The total amount of data will be about four hours. The 
corpus includes Japanese orthographic text and the corresponding alphabetic transcription and acoustic-linguistic tags.

\section{CONCLUSION}

This paper outlines various speech corpora in Japan. The present status of speech database projects in Japan is described briefly.

It also refers to the Real World Computing Program formulated by the Japanese government. The RWC Program is expected to contribute to the collection and utilization of large scale speech and text corpora.

ATR speech databases are the largest on scale and are accessible by the public. ASJ speech corpora are widely used by the speech community.

Continuous speech corpora, especially of spontaneous speech in Japan, are still in preparation. In the future, it will be increasingly necessary to enrich speech/language corpora in Japan, and to promote the collection and utilization of oriental language corpora which use non-Roman characters.

\section{ACKNOWLEDGEMENTS}

The author wishes to thank all the members of the JEIDA Speech Input Systems Subcommittee for their valuable help in data collection. He also wants to express his appreciation to the members of the Priority Area Projects of "Spoken Language," "Spoken Japanese" and "Spoken Dialogue." $\mathrm{He}$ is very grateful to all the members of the Continuous Speech Database Committee of the Acoustical Society of Japan, and of the Spoken Dialogue Corpus Working Group of the Priority Area Project "Spoken Dialogue."

\section{Related web pages for reference :}

http://www.aist-nara.ac.jp/IS/Shikano-lab/

database/internet-resource/speech_db/

http://www.tk.elec.waseda.ac.jp/speech-db/

http://www.milab.is.tsukuba.ac.jp/jnas/

http://www.isip.msstate.edu/projects/ldc jeida/jeida.html

The sites in the following list give information in Japanese. http://winnie.kuis.kyoto-u.ac.jp/taiwa-corpus/ http://www.etl.go.jp/etl/onsei/Misc/etlwd.html http://www.rwcp.or.jp/wswg/rwcdb/

\section{REFERENCES}

Fujisaki, H. (1987). “Overview of the Japanese National project on advanced man-machine interface through spoken language," Proc. First European Conference on Speech Technology, Edinburgh (Handout), 1-8.
Hayamizu, S., Itahashi, S., Kobayashi, T., and Takezawa, T. (1993). "Design and creation of speech and text corpora of dialogue," IEICE Trans. Inf. Syst. E76-D, 1722.

Itahashi, S. (1985). "Speech database of discrete words," J. Acoust. Soc. Jpn. (J) 41, 723-726 (in Japanese).

Itahashi, S. (1986). "A Japanese language speech database," Proc. ICASSP 86, Tokyo, Japan, Paper 7.4, 321-324.

Itahashi, S. (1987). " "Speech database," J. Inst. Electron. Inf. Commun. Jpn. 70, 433-438 (in Japanese).

Itahashi, S. (1990). "Recent speech database projects in Japan,” Proc. ICSLP 90, Kobe, Japan, Paper 24.1, 10811084.

Itahashi, S. (1991a). "Creating speech corpora for speech science and technology," IEICE Trans. E-74, 1906-1910.

Itahashi, S. (1991b). "Large scale Japanese dialect speech corpora," Proc. Eurospeech 91, 513-516.

Itahashi, S. (1991c). "Activities of the ASJ continuous speech database committee," Preprints ESCA Workshop on International Cooperation and Standardization of Speech Databases and Speech I/O Assessment Methods, Chivari, Italy, 1-3.

Itahashi, S. (1991d). "A noise database and Japanese common speech data corpus," J. Acoust. Soc. Jpn. (J) 47, 951-953 (in Japanese).

Itahashi, S. (1992). "Speech databases by Grant-in-Aid for Scientific Research,” J. Acoust. Soc. Jpn. (J) 48, 894898 (in Japanese).

Itahashi, S. (1998a). "Speech corpus by "spoken dialogue" Project," Proc. First International Workshop on East Asian Language Resources and Evaluation (EALREW 98), Tsukuba, Japan, 156-161.

Itahashi, S. (1998b). "On speech and text database activities in Japan," Proc. First International Conference on Language Resources and Evaluation (LREC '98), Granada, Spain.

Itahashi, S. and Sugito, M. (1990). “Collecting large scale speech corpora including many dialects - In case of the priority area project: Prosodic features of spoken Japanese," Preprints International Workshop on International Coordination and Standardization of Speech Database and Assessment Techniques for Speech Input/ Output," Kobe, Japan, 13-17.

Itou, K., Takeda, K., Takezawa, T., Matsuoka, T., Shikano, K., Kobayashi, T., Itahashi, S., and Yamamoto, M. (1998). "Design and development of Japanese speech corpus for large vocabulary continuous speech recognition," Proc. First International Workshop on East Asian Language Resources and Evaluation (EALREW 98), Tsukuba, Japan, 98-103.

Makino, S., Shirokaze T. and Kido, K. (1990). "A distributed speech database with an automatic acquisition system of speech information," Proc. ICSLP 90, Kobe, Japan, Paper 24.9, 1109-1112.

Nakajima, T., Suzuki, T., and Ohmura, H. (1973). "Data file control system for speech research," Tech. Rep. Speech Acoust. Soc. Jpn. S73-07 (in Japanese). 


\section{S. ITAHASHI : ON RECENT SPEECH CORPORA ACTIVITIES IN JAPAN}

Sagisaka, Y., Abe, M., Umeda, T., Katagiri, S., Takeda, K., and Kuwabara, H. (1990). "A large scale Japanese speech database," Proc. ICSLP 90, Kobe, Japan, Paper 24.4, 1089-1092.

Takeda, K., Sagisaka, Y., and Katagiri, S. (1987). "Acoustic-phonetic labels in a Japanese speech database," Proc. First European Conference on Speech Technology, Edinburgh, 13-16.

Takezawa, T., Morimoto, T., and Sagisaka, Y. (1998). "Speech and language databases for speech translation research in ATR," Proc. First International Workshop on East-Asian Language Resources and Evaluation (EALREW 98), Tsukuba, Japan, 148-155.

Tanaka, K., Hayamizu, S., and Ohta, K. (1986). "A demiphoneme network representation of speech and automatic labeling techniques for speech database construction," Proc. ICASSP 86, Tokyo, Japan, Paper 7.1, 309-312.

Tanaka, K., Hayamizu, S., and Ohta, K. (1990). "The ETL speech database for speech analysis and recognition research," Proc. ICSLP 90, Kobe, Japan, Paper 24.7, 1101-1104.

Tanaka, K., Hayamizu, S., Yamashita, Y., Shikano, K., Itahashi, S., and Oka, R. (1996). "Design and data collection for a spoken dialogue database in the Real World Computing (RWC) program," J. Acoust. Soc. Am. 100, Pt.2, 2759.

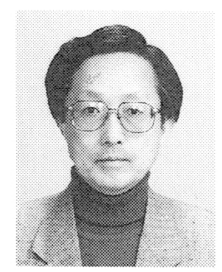

Shuichi Itahashi received the B.S., M.S. and Ph.D. degrees in electrical communication engineering from Tohoku University, Sendai, Japan, in 1964 1967, and 1971, respectively. From 1970 to June 1972 he was a Research Associate at the Research Institute of Electrical Communication, Tohoku University, where he worked on spoken word recognition utilizing linguistic information. In July 1972 he joined the research staff of the Electrotechnical Laboratory, Ministry of International Trade and Industry, Japan, where he worked on speech analysis and perception. From 1977 to 1978 he was a guest researcher at the Royal Institute of Technology, Stockholm. In 1982 he moved to the University of Tsukuba, Tsukuba, Japan. Since then he has been working on speech, image and natural language processing. He is currently a Professor of the Institute of Information Sciences and Electronics, University of Tsukuba. He has been the Dean of the College of Information Sciences, University of Tsukuba during 1994 to 1997. He has been Chairperson of the Speech Input/Output Systems Expert Committee of the Japan Electronic Industry Development Association (JEIDA) since 1981, and also of Speech Database Committee of the Acoustical Society of Japan since 1991, where he has been working with speech databases. He has been Chairperson of the Special Interest Group on Speech and Language Understanding of the Japanese Society for Artificial Intelligence since April, 1997. Dr. Itahashi is a member of the Institute of Electrical and Electronics Engineers, the Acoustical Society of America, the European Speech Communication Association, the Acoustical Society of Japan, the Institute of Electronics, Information and Communication Engineers, the Information Processing Society of Japan, the Japanese Society for Artificial Intelligence, the Association for the Natural Language Processing and the Japanese Cognitive Science Society. 